\title{
Primary Progenitor Muscle Cells for Regenerative Medicine: Standardization of Therapeutic Protocols and Optimized In Vivo Murine Model for Volumetric Muscle Loss
}

\author{
Alexis Laurent ${ }^{1}$, Nathalie Hirt-Burri ${ }^{1}$, Clotilde Amiot ${ }^{1}$, Corinne Scaletta ${ }^{1}$, Lee Ann Applegate ${ }^{1}$ \\ and Anthony S. de Buys Roessingh ${ }^{2 *}$ \\ ${ }^{1}$ Musculoskeletal System Department, Lausanne University Hospital, Switzerland \\ ${ }^{2}$ Department of Pediatric Surgery, Lausanne University Hospital, Switzerland
}

*Corresponding author: Anthony S. de Buys Roessingh, Pediatric Surgery Service, Lausanne University Hospital, CHUV, University of Lausanne, CH-1011 Lausanne, Switzerland.

Contributed equally: Alexis Laurent and Nathalie Hirt-Burri

To Cite This Article: Anthony S. de Buys Roessingh, Primary Progenitor Muscle Cells for Regenerative Medicine: Standardization of Therapeutic Protocols and Optimized In Vivo Murine Model for Volumetric Muscle Loss. 2020 - 8(2). AJBSR.MS.ID.001257. DOI: 10.34297/ AJBSR.2020.08.001257.

Received: 阱 March 13, 2020; Published: 眥 March 18, 2020

\begin{abstract}
Skeletal muscle tissue engineering constitutes an emerging therapeutic repair strategy aiming for structural and functional restoration following traumatic injury, deep burns, congenital malformation or surgical tumor removal. As for similar musculoskeletal acute and degenerative affections, allogenic progenitor cell therapy represents a promising clinical approach to synergistically supplement traditional surgical care. Preliminary studies have established the adequation of primary human fetal muscle progenitors (hFMPs) for applications in regenerative medicine. Such therapeutic cell sources combined with bioresorbable scaffolds optimally integrate in murine muscle injury models without causing immune rejection. The present work aimed at functional recovery assessment following standardized application of hFMPs in an optimized murine skeletal muscle wound model. Cryopreserved hFMPs were initiated and culture-expanded before seeding in equine collagen scaffolds. Gastrocnemius muscles of C57BL/6 mice were injured following a standardized protocol. Resulting volume defects were treated with collagen constructs yielding marked hFMPs $\left(10^{5}\right.$ cells/construct), constructs alone or remained untreated, assorted to appropriate internal controls. Histological and biomechanical analysis of muscle tissue and integrated therapeutic constructs were performed at the time of surgery and during the following 8 weeks. Both therapeutic protocols and in vivo models were optimized and standardized, based on internal experience concerning progenitor cell therapies for cutaneous reconstruction. Results indicated significantly improved function in all study groups treated with hFMPs. In particular, absolute peak twitch tensions measured on injured muscles were relatively superior in value at different time points after progenitor cell therapy application. Engraftment of hFMPs in murine wounded muscle enabled xenogeneic tissue repair stimulation and overall improvement of functional recovery. The combination of murine muscle wound model and therapeutic cell delivery method was determined as optimal for assessing muscle functional characteristic evolution notwithstanding severe tissue injury by volume loss. Such data support further translational investigation of allogenic progenitor cell therapy for human muscle defects and injuries.
\end{abstract}

Keywords: Cell therapy; Tissue engineering; Regenerative Medicine; Progenitor cells; Volumetric muscle loss; Biomechanical analysis; Murine model

Abbreviations: cATMP- combined Advanced Therapeutic Medicinal Product; DAPI- 4',6-diamidino-2-phenylindole; DMD- Duchenne Muscular Dystrophy; DMEM- Dulbecco's Modified Eagle Medium; ECM- Extracellular Matrix; EDTA- Ethylenediaminetetraacetic Acid; FBS- Fetal Bovine Serum; GMP- Good Manufacturing Practices; H\&E- Hematoxylin and Eosin; hFMPs- human Fetal Muscle Progenitors; MCB- Master Cell Bank; MTMuscle Transplantation; NCAM- Neural Cell Adhesion Molecule; PBB- Progenitor Biological Bandage; PBS- Phosphate Buffered Saline; Pt- absolute Peak twitch tension; Px- Passage Number x; RT- Room Temperature; RT ${ }^{1 / 2}$ - time for half relaxation from peak; TP- Time to Peak; VML- Volumetric Muscle Loss; WCB- Working Cell Bank 


\section{Introduction}

Traditional reconstructive surgery remains a primary practical option for extensive and profound tissue defects, as no tangible alternative consolidated therapeutic solution is currently available for effective management of these complex clinical cases. Traumatic injury, congenital malformation, burn wounds or surgical tumor removal potentially cause or lead to such significant defects, drastically impacting both structure and function of cutaneous, subcutaneous and musculature tissues. Intrinsic and relevant potential for structural tissue regeneration is inhomogenous and scarce within the human body, diminishing with progressing biological age of patients $[1,2]$. In the case of traumatic injury implicating structural predjudice, lesional extent and severity thereof dictate modalities and outcomes of muscle tissue healing. Inflammatory responses mediating macrophage invasion and satellite cell fusion intervene early in physiological repair mechanisms of skeletal muscles [3].

Generation and interplay of extracellular matrix (ECM) elements such as laminin, fibronectin, collagen and growth factors seem to play primordial roles in muscle fiber regeneration $[4,5]$. As long as quantitative skeletal muscle tissue loss does not exceed intrinsic self-repair capacities, newly formed and maturating myofibers may support muscle structural regeneration processes $[2,4]$. However, critical destructive lesions of muscle tissue drastically hinder or negate spontaneous restoration of original physiological structure and properties, leading to fibrous scarring potentially impacting and restricting contractility and general function. Significant destructive muscle injuries may be defined as volumetric muscle losses (VMLs), implying the absence by removal of tissue components such as nerves, vessels, ECM, satellite and muscle cells [6,7]. Current standards of care for VML comprise free muscle transfer (with surgical vasculature and innervation restoration) or muscular transfer flap, while protocols describing myoblast transfer therapy actively occupy translational medical research [6].

Transfer flaps are similar to donor site grafts for burn patients, allowing for rapid lesion coverage using autologous tissues and demonstrably improving strength after extensive physical rehabilitation. Nonetheless, full restoration of muscle function is not attainable within such patient management protocols [8]. Skeletal muscle tissue engineering constitutes an emerging therapeutic repair strategy aiming for structural and functional restoration possibly attained by whole-tissue replacement in various clinical applications such as muscle loss, cardiac surgery or Duchenne Muscular Dystrophy (DMD) [2,9-13]. Injections of striated muscle cells into cardiac scar tissue following myocardium infarct aided the creation of functional contractile new muscle tissue [14]. Substantiated by promising results in murine models and limited improvement in human patients, clinical trials were conducted around cell therapies for DMD, however muscle function improvement was not demonstrably ameliorated [15-18]. Marginal results were reported for muscle transplantation (MT) on one patient exhibiting $10 \%$ of muscle fibers expressing donor-derived dystrophin in a biopsy yet failing to demonstrate functional benefits [16].

Limitted efficacy led to trial discontinuations, while translational research endures in areas of regenerative medicine such as myoblast transfer therapy or progenitor cell therapy. Primary human fetal progenitor cells isolated within defined protocols are characterized by numerous intrinsic advantages conferring optimal suitability to such cell types for regenerative medicine applications. Extensive expansion potential, high proliferation rates and low technical culture requirements enable pragmatic establishment of robust cryopreserved cell banks to be used in research and therapeutic clinical settings. Additional attributes such as stable differentiated phenotypes, high resistance to oxidative stress, feeble propension towards immunogenicity or tumorigenicity and excellent overall biocompatibility strengthen the candidacy of primary progenitor cells as optimal allogeneic therapeutic agents $[19,20]$. Highly consistent and stable progenitor cell banks were developed in the Lausanne University Hospital, valorizing a single organ donation by deriving primary dermal fibroblast progenitors for clinical use. Due to thorough validation of industrialized processing of this source and progeny materials thereof, suitability of the latter for meeting stringent technical requirements of therapeutic product development was demonstrated [21].

Furthermore, clinical experience of over 20 years exists in the Lausanne Burn Center around the use of progenitor cell therapies. Progenitor Biological Bandages (PBBs) consist in dermal progenitor fibroblasts seeded on a bioresorbable collagen scaffold $(9 \times 12 \mathrm{~cm})$, which can potentially be manufactured at extensive industrial scales (> $10^{9}$ constructs/cell bank) using the same cell source [20-22]. Such constructs are used internationally for clinical investigations and treatment of ulcers, donor site grafts and second to third degree burns with unique results [23-25]. Extrapolation of fundamental and translational research performed on cutaneous structures with PBBs was sought in the domain of muscle replacement or repair, using cryopreserved stocks of primary human fetal muscle progenitor cells (hFMPs) derived as a therapeutic cell source. The scope of preliminary assessments of the latter comprised expansion and proliferation potential, stability during cryopreservation, population identity and purity, as well as in vivo integration and persistence (PKH26 marking of cells) in defined immune-competent murine models [26].

An effective delivery method was devised for hFMPs to be used in large muscle loss murine models in the form of cell-seeded three-dimentional collagen scaffolds, which allowed to ascertain preliminary safety and cell persistence in host tissues. Therefore, and in continuation of the aforementioned investigations, the present study mainly comprised analysis and standardization of bioengineered therapeutic construct delivery using an optimized murine large muscle wound model (VML model). The overall purpose was the evaluation of the effect of progenitor cell therapy using hFMPs on muscle tissue contractile recuperation potential. hFMP cell integration in collagen scaffolds and host tissues was analysed using immunohistochemistry, while evaluation of muscle 
structure and contractile function regeneration was performed using electrophysiological and biomechanical studies. This work allows for better understanding of progenitor cell therapy in the context of regenerative medicine applied to muscle tissue repair, pathing the way to a broadened translational research developmental approach of the musculoskeletal system in general. Data presented herein support further investigation of hFMP therapy for human muscle defects and injuries.

\section{Materials and Methods}

\section{hFMP culture and collagen scaffold preparation}

For the different experimental purposes of the study, primary human muscle progenitors isolated from the thigh of a male fetus (16 weeks gestational age) were made available as described previously and in accordance with the State Ethical Committee and institutional Biobank regulations [26]. The hFMPs from an established Master Cell Bank (MCB) at Passage 1 (P1) were cultureexpanded to create a Working Cell Bank (WCB) at P2. WCB vials were initiated for the experiments and cells were culture-expanded in T75 culture flasks $\left(75 \mathrm{~cm}^{2}\right.$, Nunc $\left.{ }^{\circledR}\right)$ using Dulbecco's Modified Eagle Medium (DMEM, Gibco ${ }^{\mathrm{TM}}$ ) supplemented with $10 \% \mathrm{v} / \mathrm{v}$ fetal bovine serum (FBS, $\mathrm{Gibco}^{\mathrm{TM}}$ ) in a $37^{\circ} \mathrm{C}$ humidified atmosphere under $5 \% \mathrm{CO}_{2}$.

Relative viable cell seeding density upon initiation was of $3 \times 10^{3}$ cells $/ \mathrm{cm}^{2}$. Nutrient medium was exchanged twice a week and cells were allowed to attain $90 \%$ confluency before passage. Cell viability was visually monitored and desmin immunohistochemistry (clone D33, 1:100, Dako, Denmark) was performed to verify that $>70 \%$ of the cells reacted positively to muscle precursor marking. Endpoint viable cell counts were established using Trypan Blue exclusion dye during harvest. hFMPs were labeled with the fluorescent cell membrane marker PKH26 (Red Fluorescent Cell Linker Kit, Sigma) as described previously [26]. Thereafter, $10^{5} \mathrm{hFMP-PKH26}$ marked cells were seeded on $4 \mathrm{~mm}$ punches of Tissue Fleece ${ }^{\circledR}$ equine collagen scaffold (Baxter, Germany) for 48 hours before animal experimentation. Constructs were incubated in culture medium until use and rinsed with phosphate buffered saline (PBS, Bichsel, Switzerland) before application.

\section{hFMP growth analysis on collagen scaffolds}

hFMPs were seeded on collagen scaffolds at a relative viable density of $2.5 \times 10^{3}$ cells $/ \mathrm{cm}^{2}$ and subsequently incubated in culture medium as described previously. Medium was exchanged twice a week and cells were enumerated after 5, 9, 15 and 21 days of culture. To perform enumeration, scaffolds were digested with collagenase as described previously [27]. In brief, collagen scaffolds were incubated for 30 minutes at $37^{\circ} \mathrm{C}$ in a solution of trypsinEDTA $\left(\mathrm{Gibco}^{\mathrm{TM}}\right)+$ collagenase (collagenase type II, Clostridium histolyticum, Gibco ${ }^{\mathrm{TM}}$ ) to completely degrade the collagen structure and dissociate the cells. Viable cell counts were therafter determined by manual microscopic enumeration using Trypan Blue exclusion dye. Experiments were performed in triplicate and in three experimental repetitions.

\section{Immunohistochemistry}

hFMPs were grown on round glass slides (1 $\mathrm{cm}$ diameter) and subsequently fixed with acetone for 10 minutes at room temperature (RT), rinced with PBS and stored until use in immunohistochemistry experiments. Collagen scaffolds were seeded with hFMPs $\left(10^{5}\right.$ cells $\left./ \mathrm{cm}^{2}\right)$ and incubated in culture medium for 48 hours as described previously. Constructs were therafter fixed in paraformaldehyde and embedded in paraffin. Immunochemistry for desmin and 5.1H11 (or Neural Cell Adhesion Molecule, NCAM) was performed as previously described [26]. In brief, after blocking nonspecific binding sites with normal goat serum (Dako), glass slides yielding cells or $5 \mu \mathrm{m}$ slices of paraffinembedded constructs were incubated with anti-desmin (clone D33, 1:100, Dako) or anti-5.1H11 (1:1000, Devemental Hybridoma Bank, University of Iowa, USA) antibodies for 30 minutes. After several PBS washes, slides and sections were incubated with biotinylated secondary anti-mouse anti-bodies (1:200, Vector, USA) for 30 minutes, followed by 30 minutes with a solution of aviden/biotin peroxidase (Vectastain ABC Kit, Vector). In parallel, cell-seeded construcs were also stained with standard hematoxylin and eosin (H\&E). Appropriate photographic imaging was performed using a LEICA DFC280 video imaging system. Experiments were performed in triplicate and in three experimental repetitions.

\section{Murine muscle wound model}

The animal study was properly considered and approved by the Ethics Committee of the Lausanne University Hospital under protocol \#1585. All the procedures involving animals were performed in compliance with the Swiss Federal Veterinary Office's guidelines based on the Swiss Federal Law on Animal Welfare and were approved by the Cantonal Veterinary Service. Twelve week old male C57BL/6 immuno-competent mice were obtained from Iffa Credo (France) and were kept in appropriate housing for at least one week before the experimentation began. Creation of a comparative, internally controlled and standardized VML model led to the use of muscles from both contra-lateral legs. The experimental workflow of the murine VML model is presented in Figure 1 . Sixty mice were anesthetized $(5 \mu \mathrm{L} / \mathrm{mg}$ body weight of a mix containing $500 \mu \mathrm{L} \operatorname{Rompun}^{\circledR}, 2 \mathrm{~mL} \mathrm{Ketalar}^{\circledR}$ and $2.5 \mathrm{~mL}$ PBS). Left gastrocnemius muscles were punched with a $4 \mathrm{~mm}$ diameter disc in a circular fashion in order to create a standardized injury (Figure 2A). The resulting cavity was filled either with a collagen scaffold alone (4 mm diameter) (Group $1 ; \mathrm{n}=18$ ), with nothing (Group 2; $\mathrm{n}=18$ ) or with a cell-seeded construct ( $4 \mathrm{~mm}$ diameter, yielding $10^{5}$ hFMPs) (Group 3; $\mathrm{n}=18$ ), whereas 6 mice were used as controls (Figures 1 and 2B). The contra-lateral gastrocnemius muscle was used as an internal control for each animal in order to normalize evolutive muscle contractile properties. Thereafter, the skin was closed by suturing and the colonies were appropriately maintained. Animals were housed in cages containing wood granule bedding, maintained on 12:12 h light-dark cycles and allowed free access to food and water throughout the study. All the experiments were performed 3 times (Figure 1). 


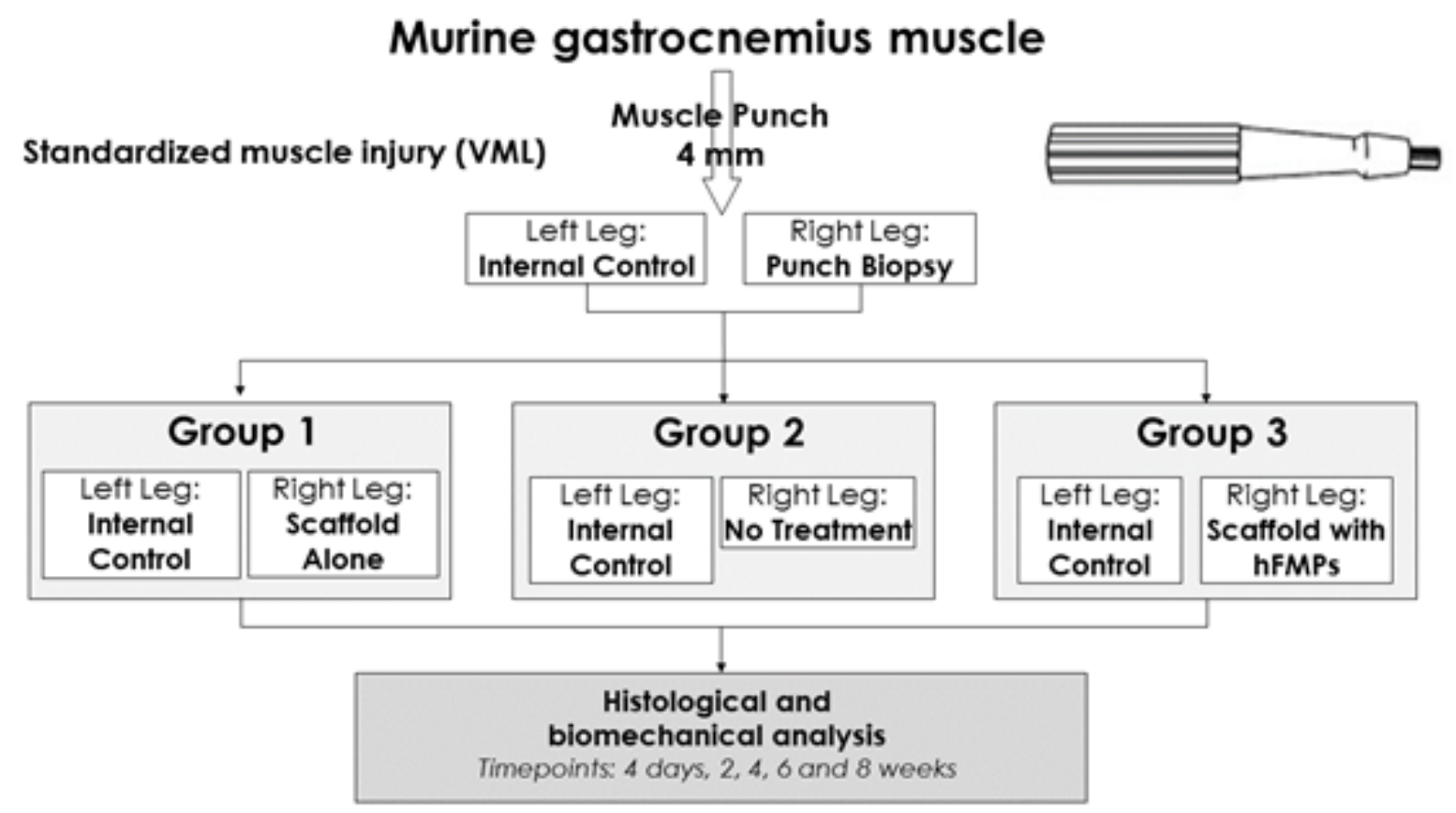

Figure 1: Experimental workflow for the murine VML model setup and comparative evaluation of hFMP-collagen construct treatment effects.
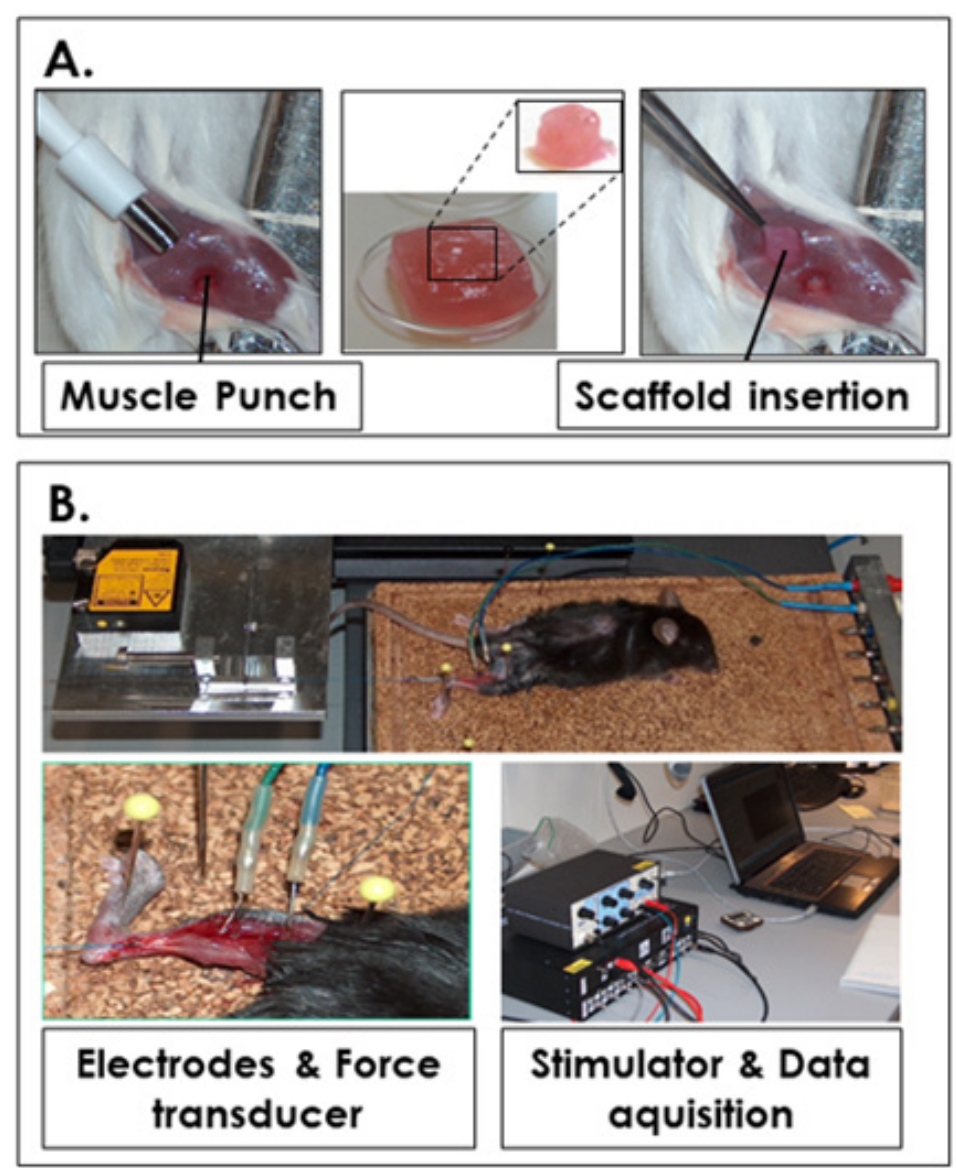

Figure 2A: Gastrocnemius muscle injury in the VML model and bioengineered construct implantation.

Figure 2B: Experimental setup for electrophysiological and biomechanical assays. 


\section{Analysis of muscle contractile properties}

For comparative muscle property recordings and analysis, animals were anesthetized by injections of a mixture of ketamine/ xylazine. Muscle strength analysis assays were performed as described previously, while tetanization and fatigue assays were secondarily investigated [13]. Murine muscle properties were characterized at timepoints of 4 days, 2, 4, 6 and 8 weeks after surgery (Figure 1). Experiments followed the protocol described hereafter. Data were recorded on a laptop with the Labview program (Figure 2B). Mice were partially shaved and the Achilles tendon of the treated hindlimb was exposed and linked to a force transducer coupled to a Watanabe Linear Corder Mark II chart recorder (WTR331). The knee joint was firmly immobilized (Figure 2B). Two fine steel electrodes were inserted into the gastrocnemius muscle. Square wave pulses of $0.5 \mathrm{~ms}$ in duration were delivered via a stimulus controller.

Stimulation voltage and muscle length were adjusted to obtain maximum isometric twitch force. The optimal muscle length was determined. Recordings started with the measurement of this optimal length between the probe and the tendon, which was then cut at its insertion on the bone (Figure 2B). A mechanical tension of 0.5 Newton was applied. A phasic twitch was then recorded and the absolute peak twitch tension (Pt), the time to peak (TTP), and the time for half relaxation from peak $\left(\mathrm{RT}_{1 / 2}\right)$ were measured. After the experiments, animals were sacrificed, gastrocnemius muscles were harvested and embebbed in Tissue-Tek O.C.T. (Sakura Finetek, Germany) medium and frozen in liquid nitrogen-cooled isopentane. Blocks were stored at $-80^{\circ} \mathrm{C}$ until further use. All the experiments were performed 3 times.

\section{Muscle tissue histology}

Cryosections of 6-10 $\mu$ m were DAPI-stained (Sigma) to visualize cellular nuclei. Sections were analyzed with an Olympus BX-40 microscope to visualize PKH26-marked cells (red marking). In order to assess hFMP cell integration, laminin immunohistochemistry was performed (L9393 antibody, 1:500, Sigma Aldrich) as described above and revealed after incubation with Alexa fluor 488 antibody (Sigma Aldrich, USA) at RT for 2.5 hours. Appropriate imaging was performed under 100X optical magnification and fluorescence excitation.

\section{Results and Discussion}

\section{hFMP growth, cell population identity and purity}
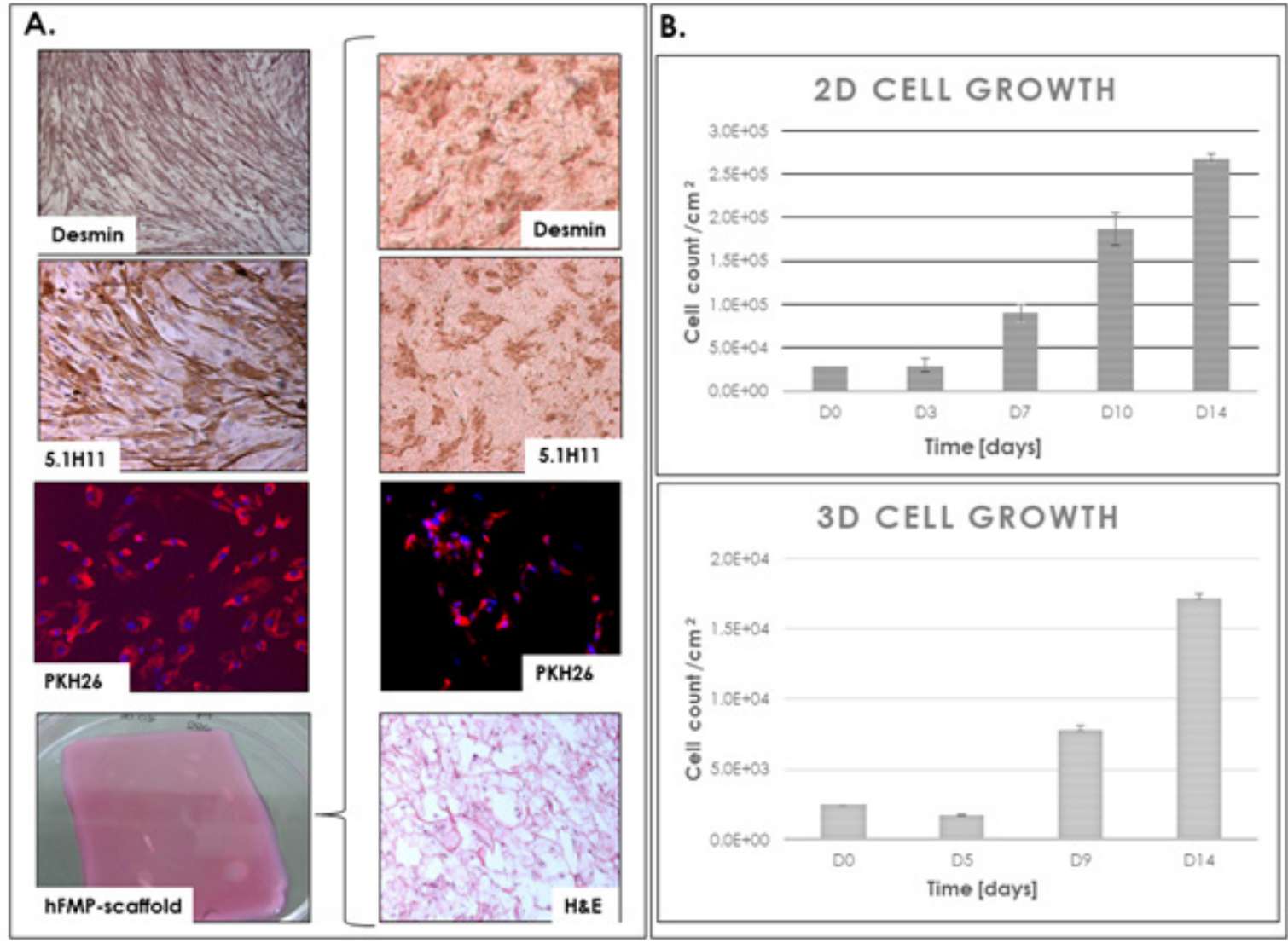

Figure 3: hFMP cell characterization

A. hFMP myogenicity characterization by immunohistochemistry (desmin and 1.5H11) and cellular marking (PKH26 and DAPI) in $2 \mathrm{D}$ monolayer culture and in cultured 3D bioengineered constructs, along with photographic representation of a hFMP-collagen construct.

B. hFMP cellular proliferation in 2D monolayer culture and in cultured 3D bioengineered constructs over two weeks. 
hFMPs from a preclinical WCB derived from a single organ donation $\left(1 \mathrm{~cm}^{3}\right.$ tissue from a male 16 weeks gestational age fetus legally and therapeutically aborted) were used in this study. At the time of cell bank establishment, hFMPs had expressed various myocyte markers such as $5.1 \mathrm{H} 11$ and desmin [26]. Conserved proliferation characteristics (cell viability, conserved morphology, population doubling values and doubling times) were observed upon initiation of cell vials, confirming initial observations and suitability of such cell types for extensive cell banking. Marker expression was reassessed after 5 years of cryopreservation and it was found that $>80 \%$ of studied hFMPs expressed $5.1 \mathrm{H} 11$ and desmin when cultured in 2D adherent monolayers or on 3D collagen scaffolds, providing capital information about cell population identity and purity evolution (Figure 3A). 2D cultures reached $90 \%$ confluency in 14 days, whereas cell counts in the collagen scaffolds increased 6-fold in the same time.

Moreover, cells displayed maintained growth in 3D (Figure 3), confirming cytocompatibility, cell colonization and integration in the collagen matrix of choice as a cell delivery system for hFMPs. Myogenic activity was observed in the scaffold based on respective 5.1H11 and desmin immunostainings (Figure 3A). PKH26-DAPI marking enabled observation of human cells integrated in the constructs (Figure 3A) by fluorescence microscopy on isolated sections. Coupled to the suitability of progenitor cell banks for thorough safety screening and applicability to good manufacturing practices (GMP) manufacturing workflows, present data confirmed hFMPs as optimal candidates for clinical cell banking at a technical level. Equine collagen scaffolds were confirmed as well adapted for therapeutic cell delivery of hFMPs, as both cyto and biocompatibility were extensively studied in the context of PBB clinical applications and results appear to be similar with progenitor muscle cells based on this study [19-22]. In addition, the use of standardized construct sizes and cell seeding densities allowed for experimental robustness as well as pragmatic use of biological materials. Indeed, the quantity of seeded cells was relatively inferior to that of comparable cell therapies, while proving sufficient after construct colonization and integration. Overall, the resulting engineered constructs were found to be relatively simple to manufacture and handle and appeared optimally designed for the intended therapeutic purpose.

\section{hFMPs for regenerative medicine and VML}

Skeletal muscle tissue engineering aiming for functional tissue replacement is based on regenerative properties of satellite cells or myoblasts, respectively their potential for proliferation and differentiation [28-30]. Limited existing therapeutic solutions prompt the development of effective protocols avoiding functional loss, volume deficiency and donor site morbidity accompanying autologous muscle transfers. Myoblast transfer therapy or skeletal myoblast transplantation represent potential treatment options for muscular dystrophies, repair of infarcted myocardium or traumatic and surgical muscle loss [31-34]. However, the effectiveness of these techniques is hindered by numerous limitations, including minimal distribution of cells after injection, immune rejection, and poor cell survival [35-43].

Optimal developmental models and therapeutic cell sources are prerequisites for sound study of significant and transposable investigational therapies. Considering cell sourcing of myoblasts or myocytes for tissue engineering, progenitor sources emerge as ideal choices as extensive clinical and industrial experience exist around such cell types [44-49]. Technical and intrinsic cellular characteristics confer numerous advantages to hFMPs destined for clinical applications, such as extensive and safe cell banking suitability, high consistency and stability, resistance to oxidative stress, potential for interplay with host ECM, paracrine modulation and immune priviledge $[19,23]$.

As demonstrated by Figure 3 and the precedent section, excellent cytocompatibility exists with clinically validated scaffolds, while cellular proliferation characteristics are conserved between 2D monolayer culture and 3D construct colonization. As for many cell therapy protocols, cell type choice proves to be the limiting factor for sound product manufacture and therapeutic success $[19,21]$. High stability and robustness of hFMPs allows for optimal integration in the engineered scaffolds and in turn in host tissues. Intrinsic immune priviledge of progenitor cells contributes to optimal histocompatibility and avoidance of effectors of the host immune system, even in a xenogeneic setting such as the present immune-competent murine model. Pragmatic medical and ethical issues are optimally covered by the use of progenitor cells, as a single organ donation after voluntary pregnancy interruption may allow to best meet the needs of millions of patients [25]. These tissues and cells are highly regulated under Transplantation Programs, which allow for clinicians to follow clear guidelines.

Considering hFMPs, one organ donation potentially yields over $10^{12}$ cells at P4 after serial culture-expansion, allowing for safe industrial-scale development of off-the-freezer therapeutic constructs. Therefore, application of such biological therapeutic agents allows for standardization of both manufacturing parameters and expected clinical outcomes.

\section{Therapeutic cell delivery for VML}

Cell delivery is a key aspect of cell therapy, as construct preparation steps and delays before clinical application may hinder cell viability or therapeutic potential [50,51]. Multiple concepts and designs of cell delivery systems have been studied for their suitability as components of bioengineered constructs, yet the limiting factor for effective product development again remains the therapeutic cell source [52-56]. In this respect and in addition to previously exposed intrinsic advantages of progenitor cells, hFMPs were observed to be outstandingly robust, as $60 \%$ cell population viability remained after 24 hours in saline solution at RT [26]. The equine collagen scaffold was chosen for hFMPs because of cyto and biocompatibility, as well as extensive experience with dermal progenitors in PBBs, which share the same design as hFMPcollagen constructs (Figure 3A). The scaffold additionally benefits from appropriate physico-chemical properties for co-application 
with biologics, adapted mechanical characteristics for implantation in skeletal muscle tissue and good acceptance from medical staff.

Cell seeding density was also derived from clinical use of PBBs, as therapeutic live cell yields have been optimized and validated for dermal progenitors, which were applied with unique results in our Lausanne Burn Center [19-24]. As it currently stands, the combination of clinical-grade therapeutic cells with a CE-marked medical device scaffold falls under the regulatory classification of a combined Advanced Therapeutic Medicinal Product (combined ATMP or cATMP). In this context, product development proves to be treacherous, emphasizing the need for a reliable biological starting material source. Pragmatic disposal of economic resources in view of regulatory approval is a prerequisite for financial viability and continuation of translational research. This objective is fully attainable by the harnessing and exploitation of progenitor cell regenerative potential.

\section{Murine VML model}

Animal models are paramount in translational therapeutic development of human muscle treatments and progenitor cells have been the focus of several in vivo studies in the past $[2,19,26,44-49]$. Low persitance of therapeutic cells in host environments is recognized and emphasizes the role of adequate functional models for treatment protocol optimization [39-43]. The use of contra-lateral legs as internal muscle controls in respective animals allowed for optimal and appropriate evaluation of relative functional muscle parameter evolution (Figure 1). The VML murine model presented herein was internally validated, as punch injuries resulted in significant and persistent functional impairment, with a relative decrease of strength $>60 \%$ between injured and uninjured muscles (data not shown) (Figure 2).

The use of punch injuries resulted in standardized injury dimensions, a prerequisite for sound comparative evaluation of muscle function impairment and therapeutic modulation thereof (Figure 2A). Experimental development and results demonstrated that the described murine large muscle injury model was well adapted for investigation of potential bioengineered therapies for muscle tissue replacement. Indeed, construct integration and functional evolution could be differentially assessed for control and treatment groups (Figure 1), yielding concrete evidence in terms of safety and efficacy for tissue repair. No evidence of immunogenicity or tumorigenicity was gathered 8 weeks postintervention, attesting to biological construct innocuity and evasion by the human progenitor cells of the murine competent immune system. Standardization of in vivo models is of great interest for the development of novel therapies and the present model allows for simple and robust evaluation of product modalities and effects with regard to relevant structural and functional endpoints.

\section{Effects of hFMP-collagen constructs on biomechanical properties of the VML model and cell persistance}

Electrophysiological and biomechanical studies allowed for optimization and establishment of a standardized murine model for VML. Engineering of hFMP-collagen constructs subsequently allowed for comparative evaluation of the effects thereof in the devised in vivo model. Electrophysiological investigation results indicated that the optimal stimuli ensuring complete muscle fiber recruitment was the application of $4 \mathrm{~mA}$ for $3 \mathrm{~ms}$ in the present experimental setup (Figures 2 and 4). For all mice, regardless of treatment group, the TTP and the $\mathrm{RT}_{1 / 2}$ mean experimental values remained statistically unchanged at all the time points tested, showing stability of the model over time in this aspect (data not shown). In addition, the absolute peak twitch tension values from the injured muscle groups were measured as lower than that of the contra-lateral controls at each time point. Comparative average rates of absolute peak twitch tension normalized to the internal control muscles at $\mathrm{t}=0,2,4$ and 8 weeks are presented in Figure 5.

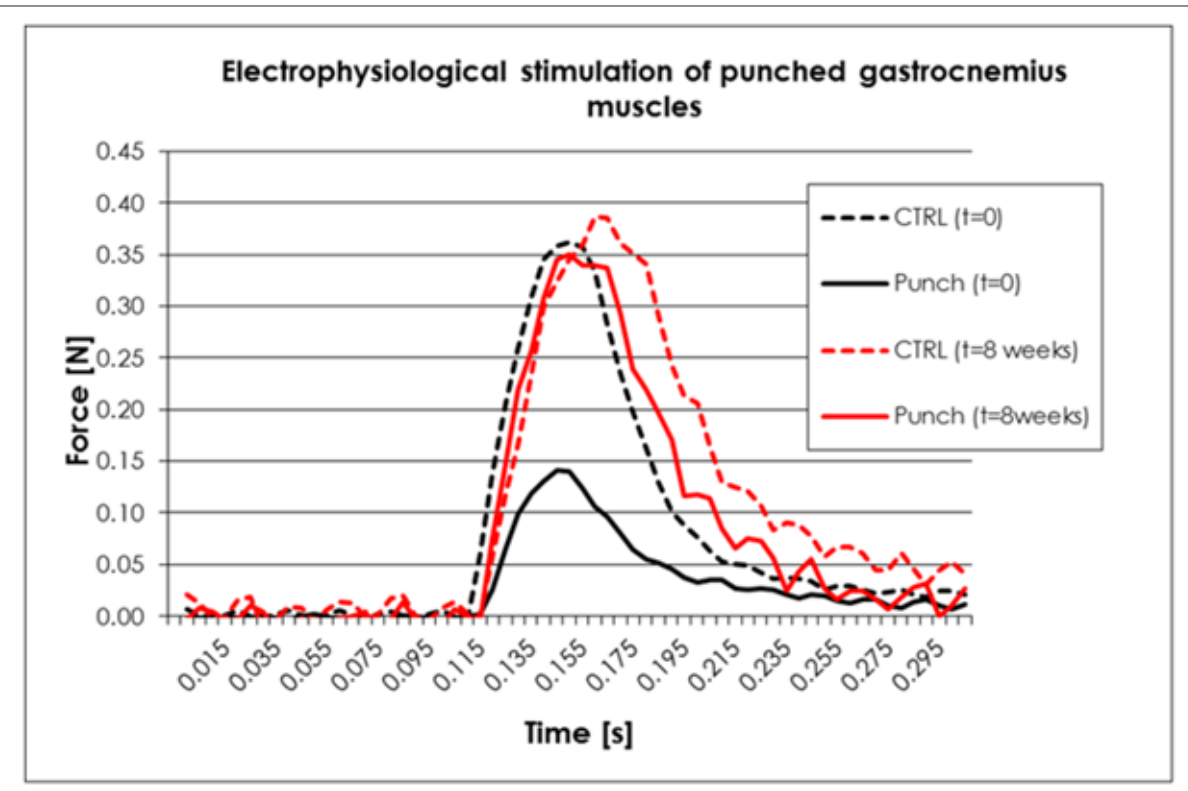

Figure 4: Comparative absolute isometric twitches encurred by electrophysiological stimulation of punched gastrocnemius muscles in the VML model at $\mathrm{t}=0$ and 8 weeks after the intervention. Stimulus parameters were $4 \mathrm{~mA}$ and $3 \mathrm{~ms}$ duration. 


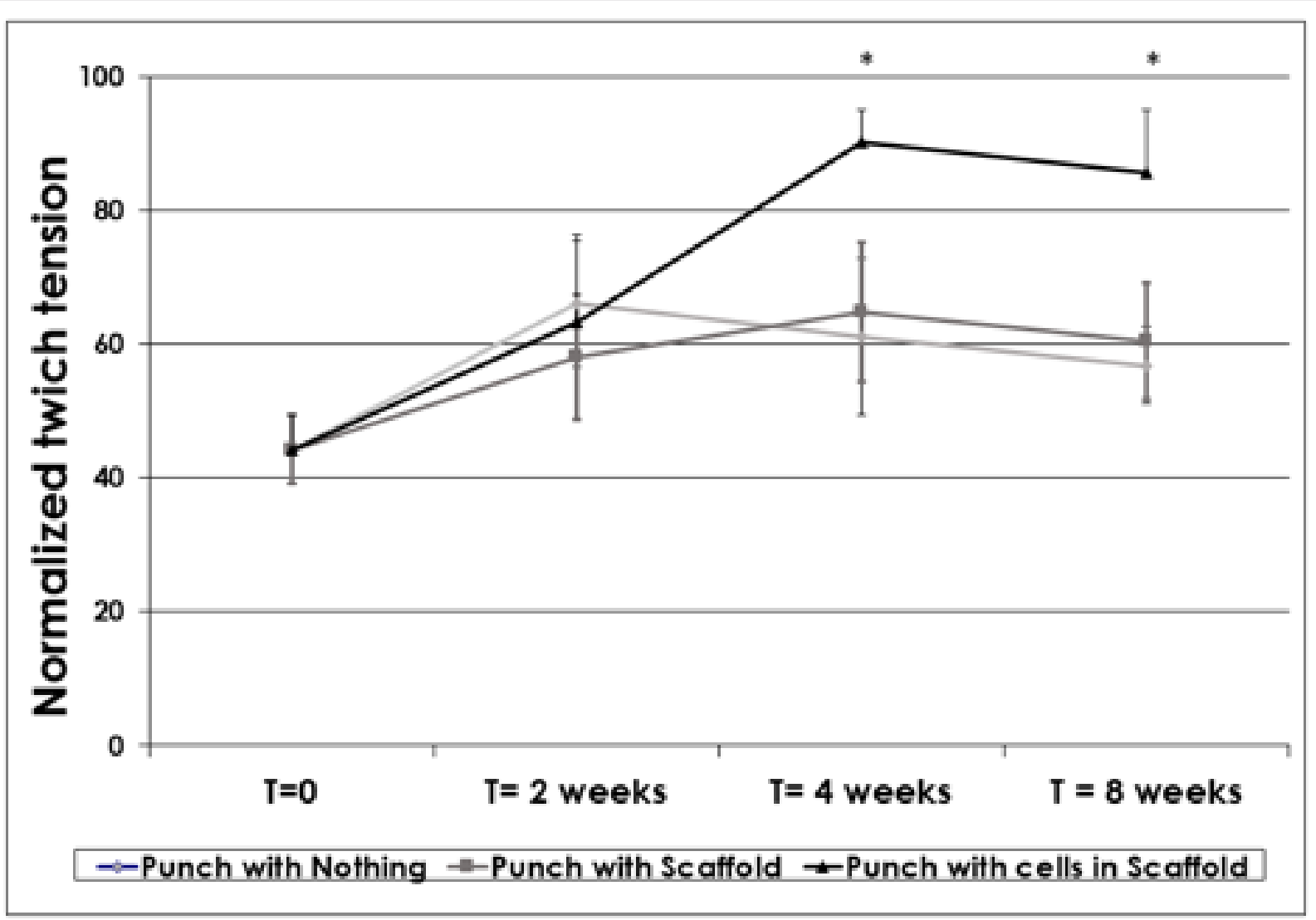

Figure 5: Comparative average rates of absolute peak twitch tension normalized to the internal control muscles for ten mice at $\mathrm{t}=0,2$ weeks, 4 weeks and 8 weeks. Statistically significant differences exist on result values at 4 and 8 -week timepoints ( $p=0.008$ and $p=0.07$ for Student's T-Test, respectively). Results are expressed as averages accompanied by standard deviations.

These biomechanical results indicated that 4 weeks postintervention, statistically significant differences were observed between the test groups, whereas relative twitch tension values for Group 3 (hFMP-collagen scaffold treatment) were superior to those of Groups 1 and 2 (scaffold alone and no treatment, respectively). Indeed, at 4 weeks, mean relative twitch tension values were determined to be 90 \% for Group 3, 65 \% for Group 1 and $61 \%$ for Group 2, respectively (Figure 5). Similar results were observed 8 weeks post-intervention, as corresponding mean values were determined to be $86 \%, 60 \%$ and $53 \%$ for Groups 3, 1 and 2 , respectively. These results indicated that from 4 weeks postintervention and on, the mice treated with hFMPs seeded on the collagen scaffolds had almost reached the maximal twich tension of the control (or $100 \%$ of relative twitch tension). Such outcomes are suggestive of near total muscle function restoration, significantly differentiating from the outcomes of comparative treatments regimens at the end of the first month post-intervention (Figure 5).

Regarding hFMP colonization and integration in implanted constructs and host tissues, immunohistochemistry enabled the observation of cell migration from the collagen scaffols towards murine muscle structures and integration over time (Figures 6 and 7). As it can be observed by non-differential DAPI staining (blue) and PKH26 marking of human cells (red) in Figure 6, hFMP cells integrated in the host muscles for up to 6 weeks, taking the place of satellite cells. The use of progenitor cells in an injured muscle seems to improve the recruitment of muscle fibers therefore improving and aiding in contractility restoration. Additional laminin marking (green) and residual PKH26 marking (red) in Figure 7 confirms human cell integration for up to 8 weeks in the murine skeletal muscle model. Histological investigations throughout the animal study did not yield evidence of immune system activation, inflammation or tumor formation around the implanted therapeutic scaffolds alone or combined with hFMPs. These aspects attest to the beneficial effects of the progenitor cells on functional endpoints of muscle repair, while xenogeneic cell presence in host tissues persists. Avoidance of host immune system activation and absence of inflammation induction by the engineered constructs deviate from reported results yielded by other muscle repair studies and accepted roles of inflammation in muscle tissue repair [57-60]. However, the functional outcomes of this study and experience around the anti-inflammatory effects of progenitor dermal fibroblasts strengthen the rationale for application of progenitor cells for optimal repair and restoration of both structural and functional properties of defective muscle tissues. 

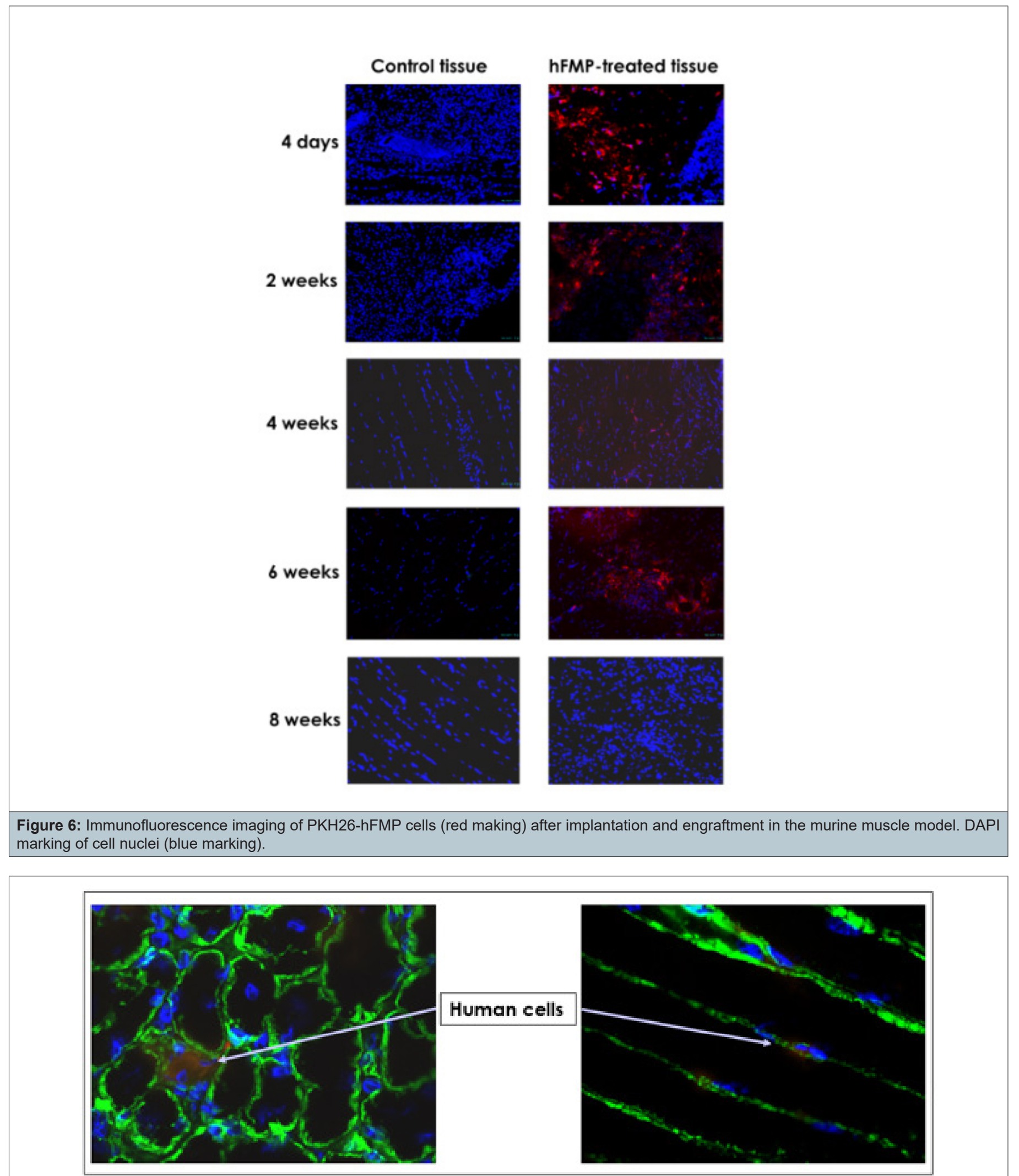

Figure 7: Laminin immunofluorescent staining (green marking) of murine muscle after 8 weeks of implantation of hFMP-collagen scaffolds, immunofluorescence imaging of PKH26-hFMP cells (red making) and DAPI marking of cell nuclei (blue marking).

\section{Conclusion}

The combination of the optimized murine VML model and standardized therapeutic cell delivery method allowed for assessing muscle functional characteristic evolution during comparative evaluation of therapeutic interventions. hFMPs were characterized and were established as legitimate candidates for 
widespread application in product manufacturing for regenerative medicine applications. Biomechanical analysis of the effects of standardized progenitor cell therapy on functional restoration of wounded muscles outlined the considerable benefits of such biologicals within translational approaches. Engraftment and persistence of human progenitor cells in the animal model were observed and illustrated both safety and immune modulation properties of the bioengineered constructs, which were able to promote and accelerate wound healing. Data yielded by this study support further investigation of hFMP therapy for human muscle defects and injuries. This work allows for better comprehension of progenitor cell therapy in the context of muscle tissue defect or injury repair and paths the way to a broadened translational research developmental approach of the musculoskeletal system in general.

\section{Acknowledgements}

We are grateful for the continued support of the S.A.N.T.E. Foundation and Sandoz Foundation. We thank the Department of Pediatric Surgery of the Lausanne University Hospital for their support for the animal experimentation, Dr André Pexieder from the School of Life Sciences from the EPFL for giving us access to the biomechanics laboratory to perform the biomechanical assays and Mrs. Judith Applegate for reviewing the manuscript.

\section{References}

1. Grasman JM, Zayas MJ, Page RL, Pins GD (2015) Biomimetic scaffolds for regeneration of volumetric muscle loss in skeletal muscle injuries. Acta Biomater 25: 2-15.

2. Passipieri JA, Christ GJ (2016) The Potential of Combination Therapeutics for More Complete Repair of Volumetric Muscle Loss Injuries: The Role of Exogenous Growth Factors and/or Progenitor Cells in Implantable Skeletal Muscle Tissue Engineering Technologies. Cells Tissues Organs 202(3-4): 202-213

3. Montarras D, Morgan J, Collins C, Relaix F, Zaffran S, et al. (2005) Direct isolation of satellite cells for skeletal muscle regeneration. Science 309(5743): 2064-2067.

4. Ciciliot S, Schiaffino S (2010) Regeneration of mammalian skeletal muscle. Basic mechanisms and clinical implications. Curr Pharm Des 16(8): 906-914.

5. Duffy RM, Sun Y, Feinberg AW (2016) Understanding the Role of ECM Protein Composition and Geometric Micropatterning for Engineering Human Skeletal Muscle. Ann Biomed Eng 44(6): 2076-2089.

6. Grogan BF, Hsu JR (2011) Volumetric muscle loss. J Am Acad Orthop Surg 19(Suppl 1): S35-37.

7. Sicari BM, Rubin JP, Dearth CL, Wolf MT, Ambrosio F, et al. (2014) An acellular biologic scaffold promotes skeletal muscle formation in mice and humans with volumetric muscle loss. Sci Transl Med 6(234): $234 \mathrm{ra} 58$.

8. Aurora A, Garg K, Corona BT, Walters TJ (2014) Physical rehabilitation improves muscle function following volumetric muscle loss injury. BMC Sports Sci Med Rehabil 6(1): 41.

9. Skuk D, Tremblay JP (2000) Progress in myoblast transplantation: a potential treatment of dystrophies. Microsc Res Tech 48(3-4): 213-222.

10. Orlic D, Kajstura J, Chimenti S, Jakoniuk I, Anderson SM, et al (2001) Bone marrow cells regenerate infarcted myocardium. Nature 410(6829): 701 705.
11. Partridge T (2002) Myoblast transplantation. Neuromuscul Disord 12(Suppl 1): S3-S6.

12. Partridge TA, Grounds M, Sloper JC (1978) Evidence of fusion between host and donor myoblasts in skeletal muscle grafts. Nature 273(5660): 306-308.

13. Dorchies OM, Wagner S, Vuadens O, Waldhauser K, Buetler TM, et al. (2006) Green tea extract and its major polyphenol (-)-epigallocatechin gallate improve muscle function in a mouse model for Duchenne muscular dystrophy. Am J Physiol Cell Physiol 290(2): C616-625.

14. Van Meter Jr CH, Claycomb WC, Delcarpio JB, Smith DM, Gruiter H, et al. (1995) Myoblast transplantation in the porcine model: A potential technique for myocardial repair. J Thorac Cardiovasc Surg 110(5): 14421448.

15. Morgan JE, Hoffman EP, Partridge TA (1990) Normal myogenic cells from newborn mice restore normal histology to degenerating muscles of the mdx mouse. J Cell Biol 111(6 Pt 1): 2437-2449.

16. Mendell JR, Kissel JT, Amato AA, King W, Signore L, et al. (1995) Myoblast transfer in the treatment of Duchenne's muscular dystrophy. N Engl J Med 333(13): 832-838.

17. Miller RG, Sharma KR, Pavlath GK, Gussoni E, Mynhier M, et al. (1997) Myoblast implantation in Duchenne muscular dystrophy: The San Francisco study. Muscle \& Nerve 20(4): 469-478.

18. Strober JB (2006) Therapeutics in duchenne muscular dystrophy. NeuroRx 3(2): 225-234

19. De Buys Roessingh AS, Hohlfeld J, Scaletta C, Hirt-Burri N, Gerber S, et al. (2006) Development, characterization, and use of a fetal skin cell bank for tissue engineering in wound healing. Cell Transplant 15(8-9): 823834.

20. Quintin A, Hirt-Burri N, Scaletta C, Schizas C, Pioletti DP, et al. (2007) Consistency and safety of cell banks for research and clinical use: preliminary analysis of fetal skin banks. Cell Transplant 16(7): 675-684.

21. Applegate LA, Scaletta C, Hirt-Burri N, Raffoul W, Pioletti D, et al. (2009) Whole-cell bioprocessing of human fetal cells for tissue engineering of skin. Skin Pharmacol Physiol 22(2): 63-73.

22. Abdel-Sayed P, Hirt-Burri N, De Buys Roessingh AS, Raffoul W, Applegate LA, et al. (2019) Evolution of biological bandages as first cover for burn patients. Adv Wound Care (New Rochelle) 8(11): 555-564.

23. Hohlfeld J, De Buys Roessingh AS, Hirt-Burri N, Chaubert P, Gerber S, et al. (2005) Tissue engineered fetal skin constructs for paediatric burns. Lancet 366(9488): 840-842.

24. De Buys Roessingh AS, Hirt Burri N, Raffoul W, Scaletta C, Applegate LA, et al. (2015) A decade after foetal skin progenitor cell therapy in pediatric burn treatment. J Regen Med 4: 1 .

25. Ramelet AA, Hirt-Burri N, Raffoul W, Scaletta C, Pioletti DP, et al. (2009) Chronic wound healing by fetal cell therapy may be explained by differential gene profiling observed in fetal versus old skin cells. Exp Gerontol 44(3): 208-218.

26. Hirt Burri N, De Buys Roessingh AS, Scaletta C, Gerber S, Pioletti DP, et al. (2008) Human muscular fetal cells: a potential cell source for muscular therapies. Pediatr Surg Int 24(1): 37-47.

27. Tenorio DM, Scaletta C, Jaccoud S, Hirt Burri N, Pioletti DP, et al. (2011) Human fetal bone cells in delivery systems for bone engineering. J Tissue Eng Regen Med 5(10): 806-814.

28. Blau HM, Webster C (1981) Isolation and characterization of human muscle cells. Proc Natl Acad Sci USA 78(9): 5623-5627.

29. Kosnik PE, Faulkner JA, Dennis RG (2001) Functional development of engineered skeletal muscle from adult and neonatal rats. Tissue Eng 7(5): 573-584. 
30. Neumann T, Nicholson BS, Sanders JE (2003) Tissue engineering of perfused microvessels. Microvasc Res 66(1): 59-67.

31. Skuk D, Roy B, Goulet M, Chapdelaine P, Bouchard JP, et al. (2004) Dystrophin expression in myofibers of Duchenne muscular dystrophy patients following intramuscular injections of normal myogenic cells. Mol Ther 9(3): 475-482.

32. Skuk D, Tremblay JP (2003) Myoblast transplantation: the current status of a potential therapeutic tool for myopathies. J Muscle Res Cell Motil 24: 287-302.

33. Skuk D, Vilquin JT, Tremblay JP (2002) Experimental and therapeutic approaches to muscular dystrophies. Curr Opin Neurol 15(5): 563-569.

34. Menasché P (2005) Skeletal myoblast for cell therapy. Coron Artery Dis 16(2): 105-110.

35. Clarkson ED (2001) Fetal tissue transplantation for patients with Parkinson's disease: a database of published clinical results. Drugs Aging 18(10): 773-785.

36. Smythe GM, Hodgetts SI, Grounds MD (2000) Immunobiology and the future of myoblast transfer therapy. Mol Ther 1(4): 304-313.

37. Beauchamp JR, Morgan JE, Pagel CN, Partridge TA (1999) Dynamics of myoblast transplantation reveal a discrete minority of precursors with stem cell-like properties as the myogenic source. J Cell Biol 144(6): 1113-1122.

38. Beauchamp JR, Pagel CN, Partridge TA (1997) A dual-marker system for quantitative studies of myoblast transplantation in the mouse. Transplantation 63(12): 1794-1797.

39. Fan Y, Maley M, Beilharz M, Grounds M (1996) Rapid death of injected myoblasts in myoblast transfer therapy. Muscle Nerve 19(7): 853-860.

40. Hodgetts SI, Beilharz MW, Scalzo AA, Grounds MD (2000) Why do cultured transplanted myoblasts die in vivo? DNA quantification shows enhanced survival of donor male myoblasts in host mice depleted of CD4+ and CD8+ cells or Nk1.1+ cells. Cell Transplant 9(4): 489-502.

41. Hodgetts SI, Spencer MJ, Grounds MD (2003) A role for natural killer cells in the rapid death of cultured donor myoblasts after transplantation. Transplantation 75(6): 863-871.

42. Qu Z, Balkir L, van Deutekom JC, Robbins PD, Pruchnic R, et al. (1998) Development of approaches to improve cell survival in myoblast transfer therapy. J Cell Biol 142(5): 1257-1267.

43. Sammels LM, Bosio E, Fragall CT, Grounds MD, van Rooijen N, et al. (2004) Innate inflammatory cells are not responsible for early death of donor myoblasts after myoblast transfertherapy. Transplantation 77(12): 1790-1797.

44. Bachoud Levi AC, Gaura V, Brugieres P, Lefaucheur JP, Boisse MF, et al. (2006) Effect of fetal neural transplants in patients with Huntington's disease 6 years after surgery: a long-term follow-up study. Lancet Neurol 5(4): 303-309.

45. Peterson CM, Jovanovic Peterson L, Formby B, Gondos B, Monda LM, et al. (1989) Human fetal pancreas transplants. J Diabet Complications 3(1): 27-34.
46. Bullard KM, Longaker MT, Lorenz HP (2003) Fetal wound healing: current biology. World J Surg 27(1): 54-61.

47. Hurst AF, Tanner WE, Osman AA (1922) Addison's Disease, with Severe Anaemia, treated by Suprarenal Grafting. Proc R Soc Med 15(Clin Sect): $19-20$

48. Sakai T, Li RK, Weisel RD, Mickle DA, Jia ZQ et al. (1999) Fetal cell transplantation: a comparison of three cell types. J Thorac Cardiovasc Surg 118(4): 715-724.

49. Sakai H, Sato T, Sakurai H, Yamamoto T, Hanaoka K, et al. (2013) Fetal skeletal muscle progenitors have regenerative capacity after intramuscular engraftment in dystrophin deficient mice. PLoS One 8(5): e63016.

50. Fan Y, Beilharz MW, Grounds MD (1996) A potential alternative strategy for myoblast transfer therapy: the use of sliced muscle grafts. Cell Transplant 5(3): 421-429.

51. Tambara K, Sakakibara Y, Sakaguchi G, Lu F, Premaratne GU, et al (2003) Transplanted skeletal myoblasts can fully replace the infarcted myocardium when they survive in the host in large numbers. Circulation. 108(Suppl1): II259-263.

52. Decary S, Mouly V, Hamida CB, Sautet A, Barbet JP, et al. (1997) Replicative potential and telomere length in human skeletal muscle: implications for satellite cell-mediated gene therapy. Hum Gene Ther 8(12): 1429-1438.

53. Urciuolo A, Urbani L, Perin S, Maghsoudlou P, Scottoni F, et al. (2018) Decellularised skeletal muscles allow functional muscle regeneration by promoting host cell migration. Sci Rep 8(1): 8398.

54. Sarrafian TL, Bodine SC, Murphy B, Grayson JK, Stover SM, et al. (2018) Extracellular matrix scaffolds for treatment of large volume muscle injuries: A review. Vet Surg 47(4): 524-535.

55. Aman M, Bergmeister KD, Festin C, Sporer ME, Russold MF, et al. (2020) Experimental Testing of Bionic Peripheral Nerve and Muscle Interfaces: Animal Model Considerations. Front Neurosci 13: 1442.

56. Ding Y, Zhao AS, Liu T, Wang YN, Gao Y, et al. (2020) An Injectable Nanocomposite Hydrogel for Potential Application of Vascularization and Tissue Repair. Ann Biomed Eng.

57. Skuk D, Roy B, Goulet M, Tremblay JP (1999) Successful myoblast transplantation in primates depends on appropriate cell delivery and induction of regeneration in the host muscle. Exp Neurol 155(1): 22-30.

58. Howard EE, Pasiakos SM, Blesso CN, Fussell MA, Rodriguez NR, et al. (2020) Divergent Roles of Inflammation in Skeletal Muscle Recovery from Injury. Front Physiol 11: 87.

59. Mosele FC, Bissi Ricci R, Abreu P, Rosa Neto JC (2020) Muscle regeneration in adiponectin knockout mice showed early activation of anti-inflammatory response with perturbations in myogenesis. J Cell Physiol.

60. Meyer GA (2018) Evidence of induced muscle regeneration persists for years in the mouse. Muscle Nerve 58(6): 858-862. 